\title{
La Perspectiva Biográfica hoy: teoría, debates, práctica
}

\author{
The Biographical Perspective today: theory, debates, practice
}

\author{
VALERIO GIANNATTASIO \\ Università degli Studi della Campania Luigi Vanvitelli \\ http://orcid.org/0000-0003-4288-9541 \\ valerio.giannattasio@unicampania.it \\ EDUARDO REY TRISTÁN \\ Universidad de Santiago de Compostela \\ https://orcid.org/0000-0002-8361-1581 \\ eduardo.rey@usc.es
}

La vida de quienes nos precedieron, la reconstrucción de sus trayectorias en ocasiones o la recuperación de los relatos de vida de modo directo o indirecto, han sido desde siempre quehacer e interés de la historia, la historia del arte, y en general las humanidades y las ciencias sociales. Pero su atención ha variado notablemente a lo largo del tiempo. De ser esencial en la reconstrucción de los fenómenos humanos del pasado durante siglos, centrada notablemente en las vidas de personajes varones destacados, pasó poco menos que al repudio una vez el foco de atención, las protagonistas de los estudios, pasaron a ser las masas, las sociedades en toda su complejidad, antes que sus reyes, héroes, mártires o santos o individuos particulares.

Esa ausencia sistemática de la historia académica, salvo excepciones ocasionales y referidas a trayectorias específicas, hizo que aquel espacio lo ocupasen literatos, eruditos o incluso aficionados. Sin desmerecer su trabajo -que a lo largo del tiempo se ha vuelto a su vez otro tipo de fuente para los estudiosos-, durante décadas asistimos a un desarrollo del género basado casi en exclusiva en el hecho, en la exaltación de los acontecimientos heroicos, o en los secretos de alcoba incluso; obras que, sin tener en muchas ocasiones ningún tipo de base metodológica ni pretensión de cientificidad, suplían lo no conocido con lo imaginado, con el posibilismo de lo probable.

La discusión estimulada por la escuela de los Annales desde finales de la década de 1920 y más adelante el cuestionamiento del estructuralismo al valor historiográfico del género biográfico, parecen haber sido definitivamente superados en las últimas tres décadas. El debate historiográfico tanto en contextos en los que persistía una fuerte tradición (como el anglosajón), como en otros en los que las historiografías nacionales habían mostrado un renovado interés por la biografía (como la española e italiana), ha provocado 
una progresiva ampliación de horizontes temáticos y epistemológicos. Todo ello permitió a la perspectiva biográfica expandirse, sobre todo en los casos en los que el uso de un enfoque multidisciplinario hacia el individuo y su tiempo fue más hábil y se orientó hacia nuevas orillas. Esa misma ampliación ha permitido que la biografía "histórica" (aquella basada en una investigación científicamente rigurosa) (re) afirmara su valor en los estudios históricos, tanto en el análisis de casos concretos como en la lectura de fenómenos y procesos - políticos, culturales, sociales, emocionales- con los cuales esos mismos casos están inevitablemente conectados y comprendidos.

La revalorización de la perspectiva biográfica ya ha sido puesta de manifiesto por especialistas en el tema como F. Dosse ${ }^{1}$ o I. Burdiel ${ }^{2}$. La "vuelta al sujeto" a la que se refiere esta autora implicó repensar el papel heurístico de la biografía, su estatuto epistemológico y sus estrategias de escritura y argumentación ${ }^{3}$. A nuestro entender, cabe destacar dos cambios clave. El primero fue el señalado por Dosse al reconocer la necesidad de acercarnos a las trayectorias personales no ya por motivos de ejemplaridad o para conocer qué había llevado a alguien a ser lo que era; sino para insertarlas en su tiempo y conocer éste a través de aquellas. Se supera con ello el relato lineal de una vida y se proponen obras que suscitan problemas, como lo serían las generadas por cualquier otra perspectiva que buscase el análisis de un tiempo y una sociedad.

El segundo cambio es el enriquecimiento que la perspectiva biográfica propone al análisis histórico y viceversa. No se trata únicamente de escribir vidas, de la biografía en el sentido absoluto que analiza Dosse al centrarse en el estudio de las grandes monografías sobre personajes individuales y tomar estas obras como unidad de análisis fundamental para diseccionar a la producción francesa o anglosajona. Nos referimos aquí al recurso al conocimiento de lo colectivo a partir de múltiples individualidades, totales o parciales: de la monografía sobre un personaje al uso de los diversos recursos que nos proporciona el utillaje metodológico del historiador: autobiografías, memorias, diarios, entrevistas, documentación personal... Todo ello tiene como eje central la experiencia del individuo; su uso, sin inscribirse necesariamente en una propuesta totalizadora de la vida de cada uno de ellos, nos permite sumar elementos en la reconstrucción de un momento histórico y en la problematización de su tiempo. La perspectiva biográfica va así más allá de la reconstrucción de trayectorias individualidades y enriquece la disciplina aportando elementos que desde lo singular permiten problematizar lo colectivo, sea eso entendido como núcleo social restringido o más amplio en términos políticos, étnicos, culturales o de genero.

Es por ello que nos parece de interés abordar nuevamente el debate sobre lo biográfico, y su relación con la historia no únicamente en la reconstrucción de trayectorias personales más o menos amplias; sino en su rol en el análisis historiográfico general.

Dosse, F., La apuesta biográfica. Escribir una vida. Valencia: Universidad de Valencia, 2007.

Véase el dosier coordinado por Isabel Burdiel en la revista Ayer 93, 2014(1).

Burdiel, I., "Presentación". Ayer 93, 2014(1): 13. 
Esta comprensión de la perspectiva biográfica esquiva en cierto modo los debates en torno a la "ilusión biográfica" (Bourdieu); lo que no obsta para reconocer su importancia en el debate que la ha fortalecido en las últimas dos décadas. No se trata únicamente de discutir si podemos reconstruir a plenitud la vida de un individuo; sino de cómo esas vidas, o fragmentos de las mismas, nos pueden ayudar a reconstruir y comprender el tiempo en que vivieron. En este sentido la relevancia del debate epistemológico se ve limitada; esto es, no debe ser elemento para condenar la perspectiva biográfica, pues la reconstrucción del pasado de un individuo, total o parcial, debe ser realizada por el historiador con herramientas, planteamientos y rigores no diferentes de los que se utilicen para la reconstrucción de procesos sociales complejos. Es en ese sentido que entendemos que la perspectiva biográfica va más allá de la reconstrucción de un personaje. Es una fórmula más, entre otras, para el conocimiento de una sociedad y un tiempo a partir de una parte de sus testimonios, pero con herramientas y presupuestos metodológicos que no tienen por qué ser más susceptibles de crítica en su rigor que los de otros enfoques. La perspectiva biográfica, por tanto, bebe de las mismas herramientas que el resto de la historiografía, no es ajena a ellas, y únicamente orienta sus fuentes a otros sujetos, en un proceso de conocimiento que comparte objetivos con cualquier otra perspectiva. A su vez, esa misma orientación y las fuentes que utiliza amplían al ángulo visual y enriquecen el conocimiento de los procesos históricos estudiados.

Al analizar la evolución de la perspectiva biográfica en la historiografía francesa, F. Dosse ya señaló que en las últimas décadas se ha dado un acercamiento a ella a partir de nuevas bases, de fórmulas renovadas que, junto con la aplicación de metodologías más rigurosas, ha permitido redescubrir el interés intrínseco del conocimiento de nuestro pasado a través del individuo, sin por ello perder la noción de lo colectivo. En este sentido es importante subrayar que la recuperación, la evolución y la profundización de la perspectiva biográfica pasa también por un cambio y una ampliación de los niveles de análisis, superando la exclusividad del personaje destacado y atendiendo también a otros actores antes olvidados, lo que significa definitivamente poner en el centro del debate la multiplicidad dimensional del ser humano. Se vuelve por tanto un relato que va de lo estrictamente personal para transformarse en un equilibrio con las varias dimensiones de lo colectivo. A su alrededor, entran en juego múltiples elementos tanto del momento histórico, social o cultural, como del propio biografiado: lo que nos cuenta de él su obra si es el caso (no solo sus datos biográficos), la imagen que generaron sus contemporáneos, la construida a posteriori... cuestiones todas que nos hablan no solo del personaje sino de la sociedad en la que vive, o la que lo evoca; y al tiempo, el carácter de la historiografía que lo recupera, el modo en el que lo hace en cada momento y sus finalidades.

El uso de lenguaje no inclusivo en las líneas previas no ha sido casualidad. El cambio en el acercamiento al género, en los últimos años, ha supuesto también la irrupción de las mujeres en él, las grandes olvidadas en otras épocas. No eran, salvo figuras puntuales y prevalentemente destacadas, objeto de interés en sí mismo, sino como mucho en función de sus personajes masculinos próximos o de la comprensión de dinámicas 
socio-culturales en las que subyace constantemente una visión masculino-céntrica. La renovación de la perspectiva biográfica y el interés de la historia, la historia del arte, y las humanidades y ciencias sociales en general, deben pasar por no seguir obviando esta carencia.

Nuestro objetivo con este dosier ha sido contribuir a la reflexión y al debate sobre la trayectoria de esta perspectiva; reflexionar acerca del significado actual de la biografía en el conocimiento de nuestro pasado; comprender nuestras sociedades en toda su complejidad -al tiempo que la propia de los procesos históricos- a través de las trayectorias individuales (una o varias de modo simultáneo en ocasiones); entender además esas trayectorias a la luz del momento en que vivieron, superando la excepcionalidad para comprendernos como hijos de nuestro tiempo con actos, logros y limitaciones únicamente comprensibles en su marco; reconocer a la multiplicidad de actores en género, raza, religión, clase o ideología, superando los esquemas que limitaron por décadas el interés a cierto tipo de actores históricos; y conocer, finalmente, qué abordajes metodológicos las diversas disciplinas ensayan actualmente en torno al género. En definitiva, una reflexión sobre el rol, las formas, los contenidos y los métodos; en torno a los logros y los aportes que ha fornido la reevaluación de la perspectiva, pero al mismo tiempo a los problemas, formales, sustanciales, epistemológicos y culturales que ha llevado consigo; en torno, por ende, al papel que ocupa la apuesta biográfica en el conocimiento de nuestro pasado así como en la reflexión sobre nuestro presente.

Dada la diversidad como factor subyacente, este dosier no pretende ser un estudio exhaustivo o definitivo sobre la perspectiva biográfica. Su ambición es ofrecer una serie de análisis que representen, a nuestro juicio, una instantánea del estado actual del género en cuestión, parte de sus estudios, de sus complejidades y de las multiplicidades que los constituyen. Los textos de los que se compone se caracterizan por tener planteamientos, ángulos y campos de estudio manifiestamente heterogéneos. En cada uno de ellos, sin embargo, podemos vislumbrar no solo la seriedad y el rigor metodológico imprescindible para un análisis escrupuloso de la vida de hombres y mujeres, sino también la importancia de la perspectiva para los distintos sectores de los estudios históricos. Precisamente a través de la lectura de los diversos escritos que componen este número, de hecho, la biografía llega a mostrarse, de alguna manera, como "necesaria" ya que es capaz de enriquecer y dar cuenta de las dimensiones cada vez más complejas de los varios campos de la investigación histórica, desde los estudios de género a la microhistoria, desde la historia social a la historia política a la oral.

A la luz de estas premisas, ordenamos los textos del dosier en dos bloques: Debates y Estudios de caso. El primero incluye los artículos que enfocan problemas o cuestiones generales, e incluye los textos que desde diferentes ángulos intentan abordar tanto las cuestiones metodológicas más generales como el estado del arte y, al mismo tiempo, dar cuenta de cómo aquellas se van a conectar con problemas específicos con respecto a los diversos campos de la investigación histórica. Se trata de escritos que intentan reafirmar la importancia de la biografía y las contribuciones que puede aportar desde un punto de 
vista historiográfico al estudio de los temas específicos a los que se refiere cada autor. El segundo bloque de artículos lo componen ensayos que, sin renunciar a abarcar el panorama general y la corriente histórica a la que se refieren, abordan el problema de la biografía en sentido más estricto, es decir, tratando experiencias de vida concretas. Los textos que integran esta sección, teniendo en cuenta los aportes y reflexiones de la historiografía más reciente y los de otras disciplinas sociales, alcanzan a ilustrar las oportunidades que la perspectiva biográfica puede brindar a su vez para la investigación histórica, llegando a partir de trayectorias individuales a proponer nuevas preguntas que pueden representar otros tantos puntos de partida para futuras investigaciones.

Precisamente la capacidad de cuestionar los "vacíos" y proponer nuevas ideas es una de las constantes que atraviesan el dosier. En este sentido, el número se abre con la estimulante reflexión de Sergio Meijide Casas sobre la posibilidad de recuperar el concepto de "biografema" (es decir, concentrarse en los detalles, gustos, inflexiones y particularidades de una vida y de los textos producidos por el biografiado) propuesto por Roland Barthes, en el ámbito de la investigación histórica sobre la biografía, y sobre cómo este ha sido utilizado, aceptado y muchas veces cuestionado por los grandes historiadores que se han ocupado del género biográfico, en primer lugar François Dosse. Meijide ilustra el contexto en el que su autor elaboró el concepto de "biografema", destacando que este nunca ha sido sistematizado definitivamente. Central en este análisis parece el discurso sobre su recepción en la obra de Dosse y sobre las perplejidades que tuvo el historiador francés hacia el "biografema", tachado de ser una mera ficción textual por dejar al lector a merced de la incertidumbre, para definir las razones por las que hasta ahora no hemos llegado a una definición orgánica de la misma noción como metodología real. El autor, por tanto, busca redimir el concepto de "biografema", especialmente por ser por definición múltiple, afirmando que puede ser utilizado con el mismo rigor que subyace al método histórico. Es más, su utilidad radica precisamente en la capacidad de poner bajo la lupa los fragmentos de las vidas, sus multiplicidades sin necesidad de recurrir a arriesgadas ficciones literarias o narrativas para tapar los inevitables vacíos que existen en la reconstrucción biográfica. En este sentido, Meijide estimula la reflexión sobre las posibilidades que una metodología ciertamente fragmentada y basada en la recuperación de los "biografemas" barthesianos puede dar tanto a los estudios estéticos como a los históricos, ya que es capaz de hacer aflorar a la superficie que, más allá de los textos y documentos, existen cuerpos y vidas que resultan a su vez compuestos, no hechos de linealidad.

Aunque diste mucho del primer trabajo, sobre todo porque está relacionado principalmente con la historia política (de la izquierda y de la mujer en México), también el texto de Verónica Oikión Solano tiene como objetivo dar un aporte importante con argumentos "modélicos" para expandir cada vez más los límites de la perspectiva biográfica. Oikión, en este caso, se centra en la necesidad de plantear una propuesta analítica para el estudio de los perfiles de las mujeres, bien como seres individuales o bien como parte de un colectivo más amplio (en este caso la izquierda mexicana de la primera mitad del siglo $\mathrm{XX}$ ). Este debe tener presentes las conexiones existentes entre la historia política, la de 
la mujer y de las relaciones de género, la historia social de género y la de las emociones; elemento este último al que la autora otorga gran respaldo (especialmente en las dinámicas entre el individuo y el grupo al que pertenece). Tal enfoque puede ofrecer un aporte fundamental para rescatar caminos biográficos que han caído en el olvido, así como la contribución del componente femenino en la esfera pública al desarrollo de la izquierda y de las redes políticas en México y más en general en el ámbito latinoamericano. Sin embargo, la propuesta de una nueva mirada biográfica debe, según la autora, superar las carencias de las biografías femeninas del siglo XX, que más que cualquier otras tienen que lidiar con vacíos documentales y tensiones constantes entre historia, omisiones y silencios. Se trata de un desafío importante para la propuesta de "reflexión analítica" sobre la biografía que necesariamente debe jugarse al borde del equilibrio entre el análisis de los recorridos individuales, los sentimientos, el compromiso colectivo y el uso de diversos campos de los estudios históricos. Sin embargo, si se realizan con rigor, los estudios biográficos basados en este enfoque podrán devolver el pasado femenino en toda su complejidad constituida por la tensión entre el actor social individual, las emociones y el sujeto histórico, conllevando un aporte innovador e importante al estudio más general de la historia política y de los movimientos políticos.

La dimensión emocional y cultural es también fundamental en el ensayo de Carolina Rodríguez-López y Antonio López Vega, quienes se detienen en la importancia que el género biográfico, precisamente en virtud de su peculiar naturaleza epistemológica, tuvo en la ampliación y profundización del campo de estudio sobre el exilio como fenómeno historiográfico. Refiriéndose al exilio español durante la Guerra Civil y tras el establecimiento de la dictadura franquista, los autores persiguen examinar cómo, gracias en cierto sentido al resurgimiento del género biográfico, se fue estudiando el exilio según la metodología propia de la historia cultural, de las emociones y de las transferencias culturales. En este sentido, los propios estudios biográficos resultaron especialmente útiles ya que permitieron analizar al exiliado como sujeto, constituido por múltiples aspectos y dimensiones, también afuera de su contexto original. Esto se debe a que el exilio, como pocas otras experiencias humanas, requiere un constante enfrentamiento con la incertidumbre interior y con la del destino de su propia tierra. Rodríguez-López y López, por tanto, ponen el énfasis en los vínculos y relaciones que se crean en el exilio y en los transfers culturales, ya que nos permiten observar a los exiliados como sujetos multidimensionales que dialogan, construyen proyectos, asimilan y transfieren elementos culturales condicionados por el contexto, y sus emociones. Todos estos elementos concurren para favorecer en las biografías de los exiliados la producción de una identidad múltiple, aún mayor que en otros sujetos históricos, en los que los caminos de ida y vuelta (reales e interiores) son decisivos. Analizar y tener en cuenta tales factores conduce a la reconstrucción de trayectorias biográficas inevitablemente únicas y compuestas, revelando una vez más la necesidad de que las biografías sean historias complejas y problemáticas en sí mismas.

Respondiendo a los estímulos que provienen de los trabajos que le preceden, el texto de Edgar de Jesús Velásquez Rivera intenta proporcionar, con un cierto perfil di- 
dáctico, unas coordenadas generales al sistematizar el amplio abanico y variedad de la perspectiva biográfica. Velásquez Rivera, a partir de la observación de la existencia de numerosas subjetividades y metodologías, esencialmente ligadas a la diversidad de enfoques y propósitos existentes en los distintos autores, propone una serie de reflexiones historiográficas sobre el desarrollo del género en la época contemporánea. Lo hace a través de los trabajos de José Luis Romero, Samuel Gajardo y León Edén, que en este contexto constituyen la base para comprender e identificar las variedades más relevantes dentro del género. A partir de estas premisas, la reflexión se expande para mostrarnos en detalle una serie de posibles nuevos "tipos" de biografía que van desde la mediática a la confesional, de la oral a la comparativa, de la crítica a los llamados desde abajo. A través de este excursus, de naturaleza explícitamente no jerárquica, el autor nos muestra las fortalezas y debilidades de cada tipo, subrayando constantemente la complementariedad de todas ellas y su carácter no excluyente. El objetivo es señalar el vasto universo en el que puede moverse un biógrafo y, por tanto, enfatizar el valor de la biografía como un recurso precioso para todas las ciencias humanas, también en virtud del carácter interdisciplinario que constituye uno de sus elementos de mayor fuerza.

El segundo bloque de trabajos lo componen estudios de caso. Mantiene una continuidad sustancial con el primero por la naturaleza problemática de los textos que lo integran, así como por la relación de intercambio recíproco entre los diferentes campos de los estudios históricos y la biografía que los ensayos proponen. Estos elementos se destacan en particular en el artículo de Andrea Alcántara Janeiro sobre Fulgencio Batista, quien con una precisa reconstrucción de la compleja relación entre biografía e historia, en la que se enfatiza la necesidad de un enfoque multidisciplinario y los aportes de otras ramas de las ciencias sociales, nos muestra una vez más la importancia que puede tener esta perspectiva en el análisis histórico; en particular, ya que esta constituye una propuesta capaz de encuadrar mejor que otras la formación y maduración de la personalidad de un sujeto, como parte del período histórico en el que se forma y vive. El análisis de la figura de Fulgencio Batista resulta considerablemente eficaz en este sentido. La autora hace especial hincapié en sus años formativos, caracterizados por el paso entre la vieja y la nueva colonialidad, para mostrar el constante entrelazamiento entre las dimensiones personales y políticas, entre los niveles micro y macro, y por lo tanto para mostrar al sujeto como producto de su contexto histórico. Esto, sin embargo, se lleva a cabo sin revelar jerarquías, tratando de colocar el camino de la vida y el contexto histórico en el que se desarrolla en un mismo nivel de importancia, en un proceso continuo de intercambio entre lo particular y lo general del cual el estudio histórico sólo puede traer beneficio.

Aunque en un escenario completamente diferente, también la trayectoria de Francesco Saverio Nitti examinada por Francesca Canale Cama puede considerarse como una manifestación de la época en que se desarrolló. En este caso, la autora destaca aspectos poco conocidos por la historiografía sobre el pensamiento y la obra del estadista italiano. Sobre todo, subraya el olvido en torno a su figura y su proyecto de relanzamiento del liberalismo italiano, luego abrumado por el fascismo y también por los principales 
componentes políticos e ideológicos del antifascismo italiano. En este sentido, la biografía de Nitti representa plenamente la crisis política e institucional que vive Italia en la delicada primera posguerra. La crisis llevará, como es sabido, al fascismo, pero en esa coyuntura no faltaron alternativas, incluidas las de corte liberal. Canale Cama nos muestra las propuestas y sensibilidades de Nitti, caracterizadas no por el egoísmo nacionalista sino por una preocupación constante por la paz continental, el desarrollo económico, la democratización y la necesidad de que la política nacional adoptara una visión europea. Se trata de un proceso de elaboración que se desarrolla sobre todo en los largos años del exilio (tema que regresa con este artículo) y luego durante la reclusión, que finalmente conduce, tras la Segunda Guerra Mundial, a la maduración de una memoria personal que correctamente puede ser considerada como transmisión de un camino colectivo en los difíciles años de entreguerras.

El olvido es también uno de los ejes temáticos del ensayo de Beatriz Peralta Peralta García, que pone en relieve los vacíos historiográficos y el aporte que el género biográfico puede proporcionar a la reconstrucción de la historia del movimiento obrero y del socialismo portugués, especialmente entre finales del siglo XIX y la fundación del Estado Novo (1933). La autora nos plantea la necesidad de estudiar no solo a figuras destacadas, proponiendo ejemplos de biografías de líderes de izquierda y del movimiento obrero que fueron muy influyentes en el Portugal presalazarista, pero que luego cayeron en la desmemoria. El objetivo es conseguir evaluar cómo ellos han influido en el desarrollo histórico. Y dado que la falta de documentos tiene en este trabajo un impacto significativo, según la autora, será especialmente necesario el recurso a fuentes orales, memoriales y fuentes secundarias. El ensayo enfatiza las considerables carencias de los estudios sobre la difusión de las ideas socialistas en el país ibérico con la escasez de biografías que refleja este mismo déficit. Sin embargo, las consideraciones y los ejemplos puestos en marcha (sobre todo el de Ernesto da Silva) sirven para corroborar la idea de que precisamente a través de la reconstrucción biográfica de figuras menores y mujeres, casi totalmente ausentes en el análisis histórico, y por tanto a través de la recuperación de obras y escritos dispersos por muchos ríos, es posible propiciar una contribución decisiva al estudio del movimiento obrero portugués entre los siglos XIX y XX.

Jorge Albeiro Holguín Pedroza, nos lleva al otro lado del Atlántico para abordar una vez más trayectorias de vida que pertenecen a la izquierda, en este caso colombiana. La obra de Holguín pone bajo observación las vidas de Ignacio Torres Giraldo y Juan de Dios Romero durante la década de 1930, el primero líder reconocido del Partido Comunista de Colombia y el segundo personaje menos considerado y a menudo presentado de manera negativa por la historiografía comunista. El texto abraza plenamente los estímulos más recientes del género biográfico, basándose en gran parte en la historia social de la izquierda, pero centrándose principalmente en las oportunidades que pueden surgir del uso de la biografía comparada como herramienta investigadora capaz de aprovechar el diálogo multidisciplinar y mostrar al conflicto social como "vaso comunicador de las historias paralelas de la izquierda Latinoamericana". A través de la reconstrucción de las experien- 
cias de los dos políticos sudamericanos, Holguín destaca no solo la dimensión ideológica sino también las relaciones, la formación y los conflictos para comprender cómo han influido materialmente en la elaboración de las formas de lucha y de asociación. En este sentido, la vida de los protagonistas define dos itinerarios que, partiendo de lo particular, nos muestran claramente las características y evoluciones de dos de las principales corrientes del marxismo colombiano, una más fuertemente ligada al leninismo y al partido y la otra más heterogénea, que privilegió el sindicalismo con referencias a Proudhon y Bakunin. En esta perspectiva, el uso de la biografía comparada se vuelve particularmente útil porque la multiplicidad de trayectorias analizadas permite mirar las experiencias políticas en una dimensión más amplia que puede dar cuenta de contradicciones y omisiones, a menudo perpetradas por historiografías partidistas o hegemónicas.

A las trayectorias ligadas al mundo de la izquierda se contrapone el estudio de $\mathrm{Ca}$ millo Robertini, que cierra el número monográfico. De hecho, el texto examina la experiencia de un joven sindicalista peronista de extrema derecha desde la segunda mitad de la década de 1950 hasta la de 1970. Lo que distingue al texto de Robertini, sin embargo, no es solo la reafirmación de un campo de estudio, el de las derechas, en continua expansión, sino también el uso explícito de la historia oral y la entrevista como instrumento de investigación histórica y composición biográfica. A través de estas herramientas, el autor pone en el centro de la atención no solo la actividad de un sindicalista, sino también su trayectoria de vida y como persona común, para recuperar su memoria, subjetividad e imaginación. El hilo conductor es la construcción de la identidad a partir del origen inmigrante del protagonista y los pensamientos de sus padres, para pasar a su formación política y a la explicitación de la acción violenta contra las formaciones marxistas en los años setenta, que bien puede insertarse en un contexto político más amplio nacional e internacional. La biografía de un militante, a partir de un nivel micro, permite así ver la evolución de las derechas y la construcción del imaginario reaccionario de los sesenta y setenta en la Argentina. Este es un análisis explícitamente parcial, cuyo propósito, sin embargo, no es ser exhaustivo. De hecho, el autor quiere intentar esclarecer una de las muchas lagunas presentes en el campo de la historia reciente Argentina (y no solo), invitándonos a aceptar el desafío de mirar el estudio de la última parte del siglo XX a partir del mundo de las derechas y de las culturas políticas que esta produjo.

Este dossier, finalmente, quiere mostrar no solo la validez científica, a esta altura (re) consolidada, de la perspectiva biográfica como género histórico, sino también su carácter interdisciplinario, su vivacidad y su eclecticismo. No es tarea nuestra responder a través de los textos presentados a dudas teóricas y metodológicas que seguirán existiendo y a las cuales solo se podrá contestar trabajando con rigor científico, independientemente del enfoque que se haya elegido o del campo de estudio de cada autor/a. En definitiva, lo que hemos tratado ha sido proponer diferentes interpretaciones que resalten la importancia de la perspectiva biográfica para la historiografía y al mismo tiempo las oportunidades y problemas que puede presentar para los diversos sectores de las ciencias sociales y de la historia en particular. 